\title{
Ultrasound and LED-based photoacoustic system for preclinical imaging of liver fibrosis
}

Kalloor Joseph, Francis, Steenbergen, Wiendelt, Bansal, Ruchi

Francis Kalloor Joseph, Wiendelt Steenbergen, Ruchi Bansal, "Ultrasound and LED-based photoacoustic system for preclinical imaging of liver fibrosis," Proc. SPIE 11642, Photons Plus Ultrasound: Imaging and Sensing 2021, 116422I (5 March 2021); doi: 10.1117/12.2577720 


\title{
Ultrasound and LED-based Photoacoustic System for Preclinical Imaging of Liver Fibrosis
}

\author{
Kalloor Joseph Francis ${ }^{\mathrm{a}}$, Wiendelt Steenbergen ${ }^{\mathrm{a}}$, and Ruchi Bansal ${ }^{\mathrm{b}}$ \\ a Biomedical Photonic Imaging group, Technical Medical Center, University of Twente, The \\ Netherlands \\ b Medical Cell BioPhysics (MCBP), Technical Medical Center, University of Twente
}

\begin{abstract}
Histopathological analysis remains a gold standard in preclinical research, which can only be performed as an end-stage analysis. In small animal studies, in order to improve the outcome, it is important to study the disease progression and treatment response over time without sacrificing animals at different time points thereby reducing the number of animals. Photoacoustic imaging with its ability to probe endogenous chromophores in the tissue and being non-invasive has great potential in this aspect. In this work, we aim to use ultrasound and photoacoustic imaging for assessment of liver fibrosis, in particular, to monitor the disease progression. We have used ultrasound and LED-based photoacoustic (US/LED-PA) imaging system, both in its handheld and tomographic mode. In the tomographic mode, the transducer and LED arrays were scanned around the animal for imaging. The imaging was performed on healthy (control) mice and mice with carbon tetrachloride (CCl4) induced liver fibrosis. The control and fibrotic mice were followed over a duration of eight weeks using US/LEDPA imaging. The animals were euthanized, imaged and histopathological analysis were performed at the end of 8-weeks. An increase in photoacoustic contrast and ultrasound echogenicity were observed progressively in the fibrotic livers compared to the control livers. The histological analysis also validated the severity of the fibrosis in the mice. The proposed approach using longitudinal monitoring of disease progression using US/LEDPA imaging can improve the outcome of the study. Since the LED-based system is compact, eye-safe, and easy-to-use, it can be of great benefit in small animal research.
\end{abstract}

Keywords: Photoacoustic, ultrasound, tomography, liver fibrosis, small animal, Medical and biological imaging

\section{INTRODUCTION}

With more than one million deaths every year worldwide, liver fibrosis is one of the major causes of mortality. ${ }^{1}$ Liver fibrosis can be due to alcoholic injury, metabolic disease, toxin, autoimmune disease, or other factors that have become common in the recent past. ${ }^{1}$ Thus there is an increasing need for the development of new drugs. However, most of the small animal studies rely on the endpoint histopathological analysis as a goal standard for the study outcome. ${ }^{2}$ Variability within the study group and differences in disease progression can considerably affect the study and thus delay the translation of new drugs. Imaging methods play an important role in this context to monitor the disease progression and treatment over time to improve the efficacy of the study. ${ }^{3}$

Imaging methods such as ultrasound (US), Computed Tomography (CT), Positron Emission Tomography (PET), and Magnetic Resonance Imaging (MRI) are also in use for preclinical imaging. ${ }^{3}$ US imaging is a noninvasive method. However, quantifying the disease stage can be difficult using the US, being a structural imaging method. PET and Micro CT can be used for preclinical imaging, but the ionizing radiation used in these modalities hinders longitudinal monitoring. On the other hand, micro MRI systems are costly and difficult to house in a small animal facility. Hence there is a need for a compact, noninvasive, functional imaging method for preclinical applications. ${ }^{4}$ Photoacoustic (PA) is a new modality in which pulsed light excites ultrasound waves from optical absorbers in the tissue. ${ }^{5,6}$ The capable of PA to provide optical contrast at ultrasound

Further author information:

K. J. Francis.: E-mail: f.kalloorjoseph@utwente.nl, Telephone: +31534891559

Photons Plus Ultrasound: Imaging and Sensing 2021, edited by Alexander A. Oraevsky

Lihong V. Wang, Proc. of SPIE Vol. 11642, 116422I · C) 2021 SPIE

CCC code: $1605-7422 / 21 / \$ 21 \cdot$ doi: $10.1117 / 12.2577720$

Proc. of SPIE Vol. 11642 116422I-1

Downloaded From: https://www.spiedigitallibrary.org/conference-proceedings-of-spie on 14 Jul 2021 
resolution enabling functional and molecular imaging. Further, the use of US transducers for detection has an added advantage to have coregistered US imaging for structural information. PA imaging has been reported in the past on mice liver fibrosis model from our group ${ }^{7}$ and using rat by Karmacharya et. al. ${ }^{8}$ An increase in PA signal contrast and oxygen saturation were reported in the fibrotic liver compared to the control.

Using LEDs as the light source for PA imaging can reduce the system cost and size, and it is eye-safe. ${ }^{9}$ Recently, LED-based PA imaging has attracted several applications, including preclinical small animal imaging. Small animal imaging is an ideal application for LED-based PA system as the imaging depth required is typically less than $3 \mathrm{~cm}$. Several applications were reported, including tumor imaging in the recent past. ${ }^{10,11}$ With multi-wavelength LEDs, oxygen saturation and imaging contrast agents were also reported. ${ }^{12,13}$ Most of the works used a handheld probe with a linear transducer array. However, a limited view of the target resulting from detection using a linear array is an issue in this configuration. To overcome the limited view problem, we developed a tomographic system. ${ }^{14-16}$

In this work, we present small animal imaging using tomographic configuration. Specifically looked at the fibrosis model to imaging the liver inside the animal. Further, we image the isolated liver using a handheld probe. Histopathological analyses were performed to confirm the fibrosis in the liver.

\section{MATERIALS AND METHODS}

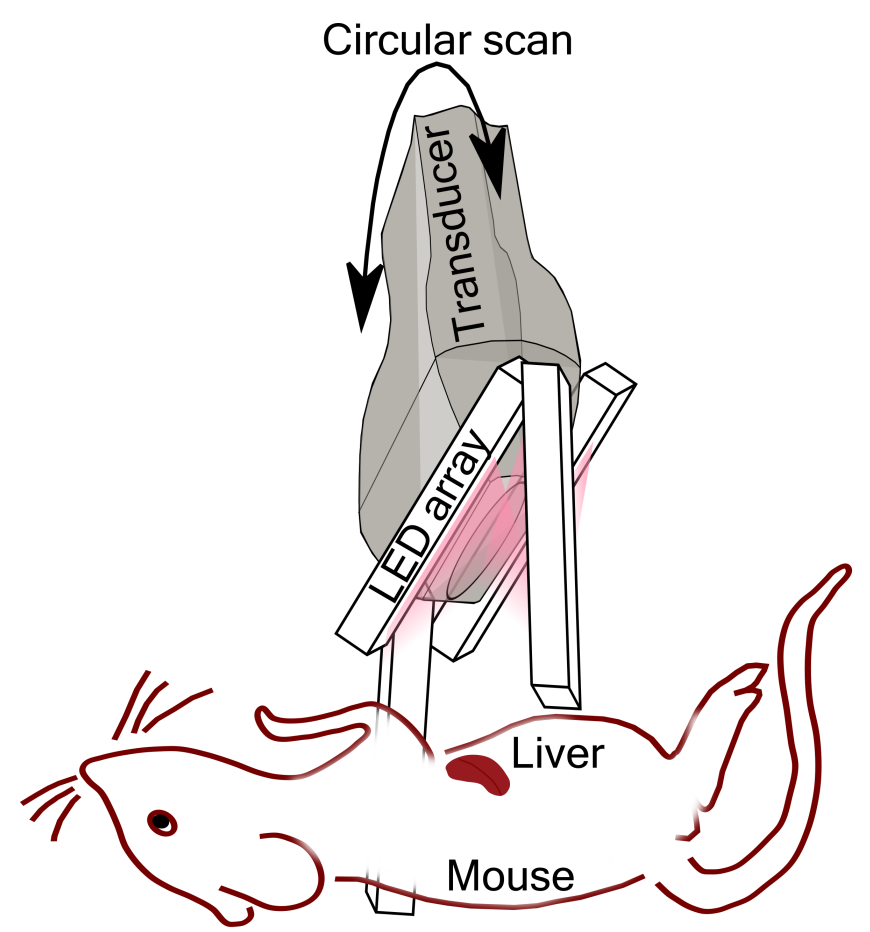

(a)

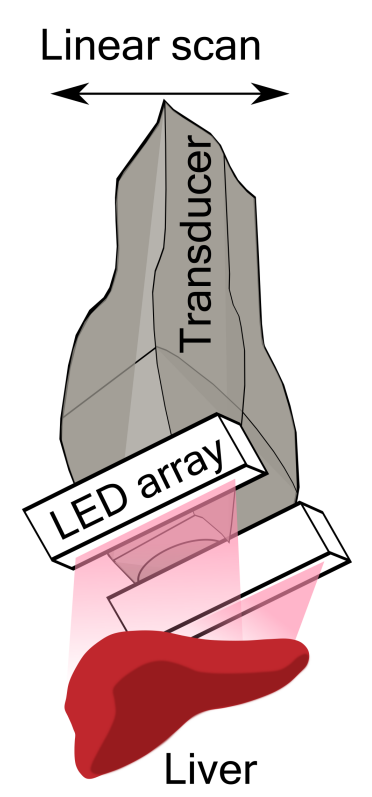

(b)

Figure 1. Liver imaging using LED-based photoacoustic system: (a) Imaging liver region of a mouse using the tomographic system using circular scan. (b) Imaging isolated liver using linear scan using a handheld probe.

In this work, we used an LED-based PA and US system AcousticX (Cyberdyne Inc., Japan). A linear transducer array of 128 elements, with a center frequency of $7 \mathrm{MHz}$ and $80 \%$ bandwidth was used for US imaging and PA detection. An LED array $(36 \times 4)$ of $850 \mathrm{~nm}$ wavelength, with a pulse energy of $200 \mu \mathrm{J}$, and a pulse duration of $70 \mathrm{~ns}$ was used as the light source. In the tomographic configuration, four LED arrays were used and two in the handheld configuration. In the tomographic configuration, two LED arrays were placed on either side of the transducer at an angle of $30.8^{0}$ with the imaging plane and two more in the imaging plane at an angle of $105^{0}$ to the transducer as shown in Fig. 1. Figure 1 shows the two imaging cases from two separate animal study. The mice were imaged using the tomographic configuration around the liver region. The isolated liver 
was imaged using the handheld probe with a linear scan to image the whole liver.

In these proof-of-concept studies, male Balb/c mice were used. The animal experiments were carried out according to the ethical guidelines for the Care and Use of Laboratory Animals (The Netherlands). A normal chow diet and water with $12 \mathrm{~h} / 12 \mathrm{~h}$ light and dark cycle were given to all the animals. Liver fibrosis was induced by injecting carbon tetrachloride $\left(\mathrm{CCl}_{4}\right)$. In the first study, two animals were used. One of the animals was given $1 \mathrm{ml} / \mathrm{kg}$ of $\mathrm{CCl}_{4}$ dissolved in olive to induce fibrosis, while the other animal used as the control was given olive oil. Both the animals were imaged at week 8 after the injection. Before imaging, the mice were euthanized and hair in the abdominal area was removed. PBS (1X) solution was used as the medium for acoustic coupling. Three angular views of $20^{0}$ steps were used in the tomographic system to image the liver region of the mice. In the second study, animals were imaged at weeks 4,6 , and 8 . The isolated liver samples were imaged using the handheld probe with a linear scan obtaining images at every $1 \mathrm{~mm}$ for a scanning area of $20 \mathrm{~mm}$. After the imaging, the liver samples were fixed with cryomatrix in isopentane and further sectioned for immunohistological analysis. Collagen I antibody was used as a fibrosis marker for histological analysis.

\section{RESULTS AND DISCUSSION}

In the first experiment tomographic imaging of a control and a fibrotic mice were performed using spatial compounding of three angular views. Figure 2 shows the tomographic imaging of control and fibrotic mice. The overlaid US and PA images of control liver (Fig. 2a) and fibrotic liver (Fig. 2b) shows the difference between the two cases. Figure 2c,d and e show the US, PA and histology image respectively of the control liver and Figure $2 \mathrm{f}, \mathrm{g}$ and $\mathrm{h}$ that of the fibrotic liver.

The green line in the figure marks the liver region. The difference in control and fibrotic liver can be observed in
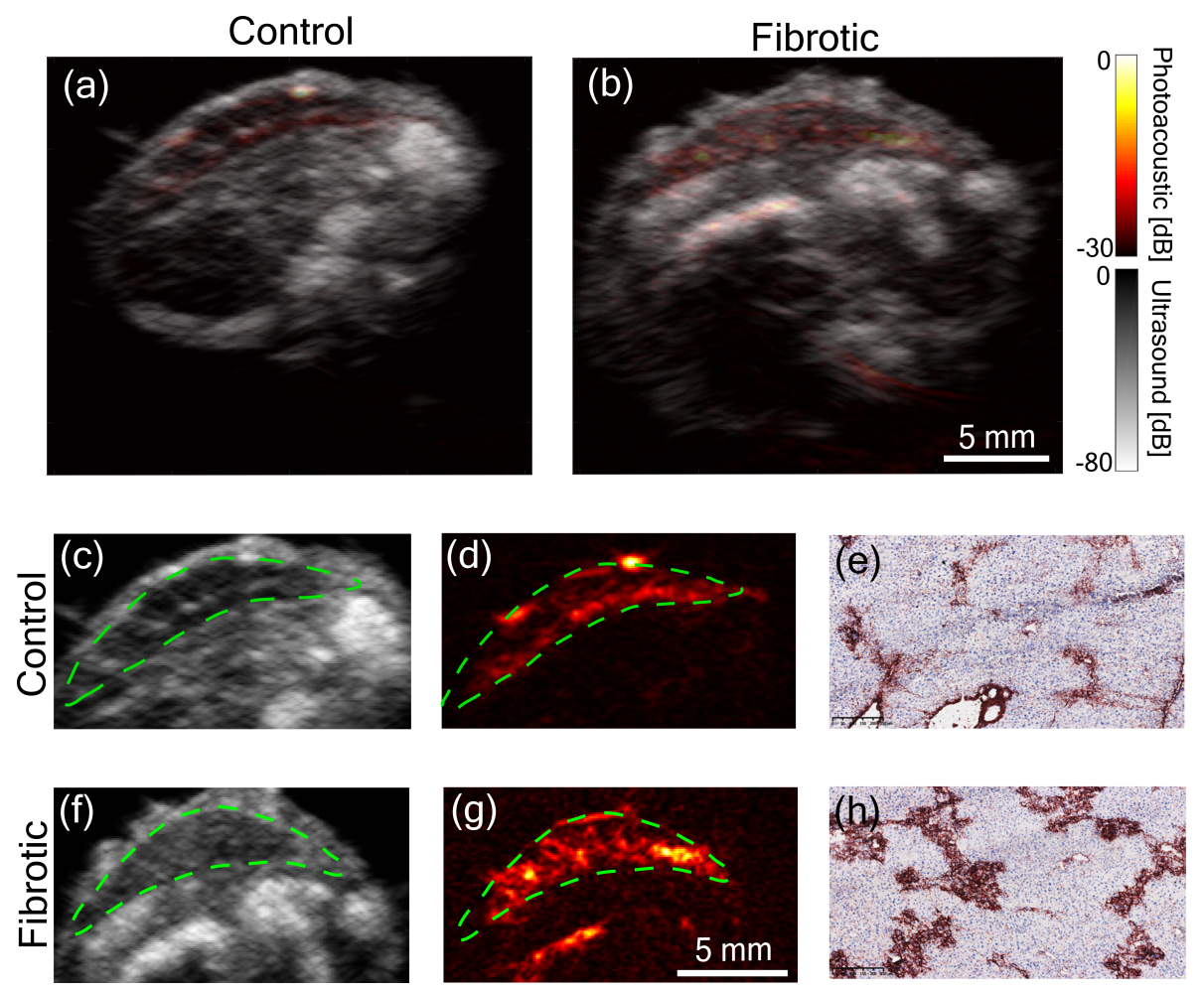

Figure 2. Small animal liver imaging. Overlaid photoacoustic and ultrasound tomographic image of (a) control and (b) fibrotic animals showing the liver region. (c, f) ultrasound, (d, g) photoacoustic, and (e, h) are histology images of control and fibrotic livers, respectively.

the US image with an increase in echogenicity in the fibrotic case. The mean pixel value in the liver US images was calculated to quantify the echogenicity. The control liver had a mean of $0.13 \pm 0.02$ while for the fibrotic liver it was $0.25 \pm 0.03$. The heterogeneity in the US liver image was estimated using the variance. In the control case, 
a variance of $0.52 \pm 0.11$ compared to $0.85 \pm 0.10$ in the fibrotic case was observed. These metrics clearly show the difference between control and fibrotic liver with US imaging. The echogenicity and heterogeneity observed in the US might be due to the hepatic nodularity developed in fibrotic liver. ${ }^{17}$ Further, PA images in Fig. $2 \mathrm{~d}$ and $\mathrm{g}$ shows significent differnce in PA contrast. A contrast value of $0.48 \pm 0.08$ was observed for the control liver in comparison to that of $0.69 \pm 0.08$ for the fibrotic case. As the PA imaging used a light of $850 \mathrm{~nm}$ wavelength the contrast is primarly from absorption of blood. Neovasculature in hypoxic liver is considered to be the primary factor contributing to this increase in PA contrast in the fibrotic liver. Figure 2 (e) and (h) show histology images with collagen I immuno-staining of control and fibrotic liver sections respectively. The fibrotic liver histology in Fig. $2 \mathrm{~h}$ shows and increased level of collagan bridges in comparison to the control liver, Fig. 2e. The increase in collagen bridges is proportional to the severity of fibrosis due to the accumulation of the extracellular matrix protein.

In the second experiment, isolated livers with progressive levels of fibrosis were imaged. Figure 3 shows the US
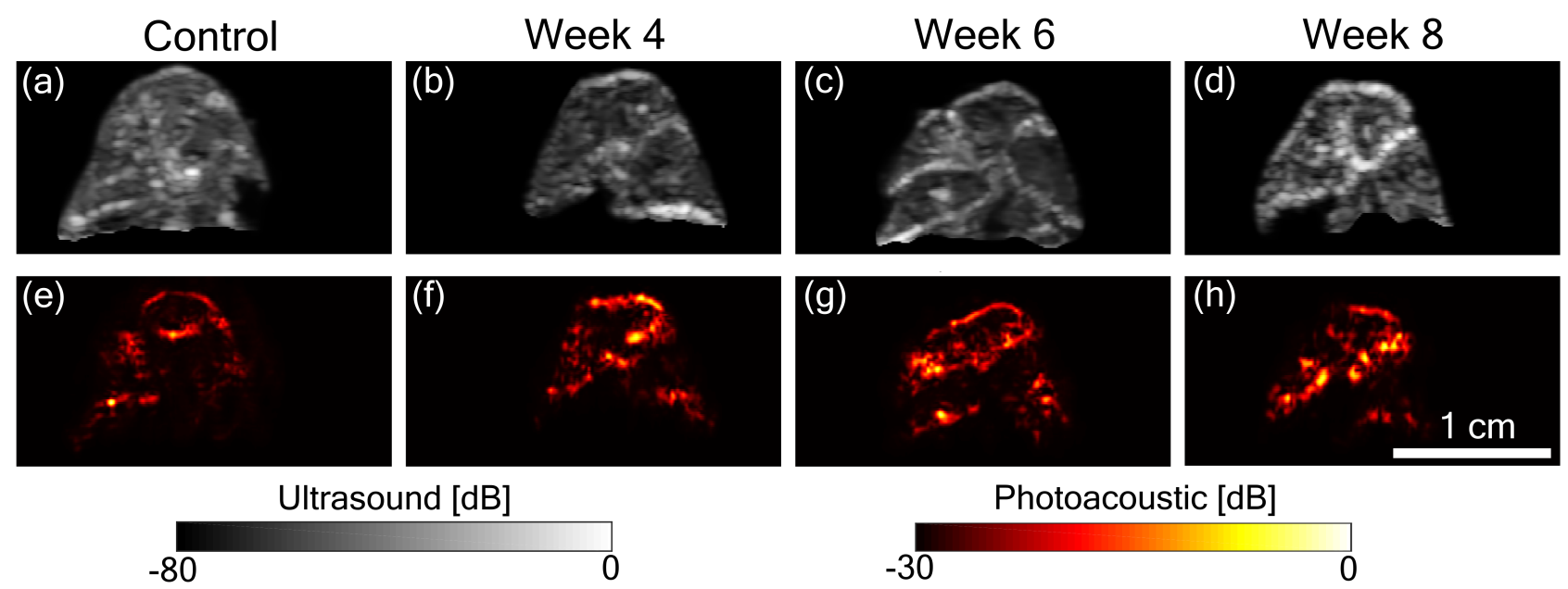

Figure 3. Photoacoustic and ultrasound images of isolated livers with fibrosis progression. Ultrasound images of livers from (a) control, (b) week 4, (c) week 6 , and (d) week 8 through $\mathrm{CCl}_{4}$ treatment. Photoacoustic images from (a) control, (b) week 4, (c) week 6 , and (d) week 8 through the $\mathrm{CCl}_{4}$ treatment.

and PA images of livers at 4, 6 , and 8 weeks of $\mathrm{CCl}_{4}$ treatment compared with the control case. The US images show an increasing level of wall thickness and heterogeneity in the liver with fibrosis progression. PA image in the control case shows a homogeneous signal from the wall and considerably less signal inside the lobes. While in the fibrotic case, an irregular wall and uneven texture with large structures inside each lobe was observed. An overall increase in PA contrast can also be observed with progress in fibrosis. These results are also in line with our previous small animal liver fibrosis study. ${ }^{7}$

\section{CONCLUSION}

The results show the possibility of a compact, low-cost tomographic imaging system for preclinical small animal studies. The clear difference between control and fibrotic liver indicates the applicability of the system for liver fibrosis studies. Further, images at different time points in the study is a promising first step for considering photoacoustic imaging for small animal liver fibrosis studies in the future.

\section{ACKNOWLEDGMENTS}

We acknowledge funding from the $4 \mathrm{TU}$ federation in the precision medicine program.

\section{REFERENCES}

[1] Koyama, Y., Brenner, D. A., et al., "Liver inflammation and fibrosis," The Journal of clinical investigation 127(1), 55-64 (2017). 
[2] Andersen, M. L. and Winter, L. M., "Animal models in biological and biomedical research-experimental and ethical concerns," Anais da Academia Brasileira de Ciências 91 (2019).

[3] Xia, J. and Wang, L. V., "Small-animal whole-body photoacoustic tomography: a review," IEEE Transactions on Biomedical Engineering 61(5), 1380-1389 (2013).

[4] Erfanzadeh, M. and Zhu, Q., "Photoacoustic imaging with low-cost sources; a review," Photoacoustics 14, $1-11(2019)$.

[5] Xu, M. and Wang, L. V., "Photoacoustic imaging in biomedicine," Review of Scientific Instruments 77(4), 041101 (2006).

[6] Attia, A. B. E., Balasundaram, G., Moothanchery, M., Dinish, U., Bi, R., Ntziachristos, V., and Olivo, M., "A review of clinical photoacoustic imaging: Current and future trends," Photoacoustics 16, 100144 (2019).

[7] van den Berg, P. J., Bansal, R., Daoudi, K., Steenbergen, W., and Prakash, J., "Preclinical detection of liver fibrosis using dual-modality photoacoustic/ultrasound system," Biomedical optics express 7(12), 5081-5091 (2016).

[8] Karmacharya, M. B., Sultan, L. R., Kirkham, B. M., Brice, A. K., Wood, A. K., and Sehgal, C. M., "Photoacoustic imaging for assessing tissue oxygenation changes in rat hepatic fibrosis," Diagnostics 10(9), 705 (2020).

[9] Zhu, Y., Feng, T., Cheng, Q., Wang, X., Du, S., Sato, N., Yuan, J., and Kuniyil Ajith Singh, M., "Towards clinical translation of led-based photoacoustic imaging: a review," Sensors 20(9), 2484 (2020).

[10] Xavierselvan, M., Singh, M. K. A., and Mallidi, S., "In vivo tumor vascular imaging with light emitting diode-based photoacoustic imaging system," Sensors 20(16), 4503 (2020).

[11] Joseph, F. K., Xavierselvan, M., Singh, M. K. A., Mallidi, S., Van Der Laken, C., Van De Loo, F., and Steenbergen, W., "Led-based photoacoustic imaging for early detection of joint inflammation in rodents: Towards achieving 3rs in rheumatoid arthritis research," in [Photons Plus Ultrasound: Imaging and Sensing 2020], 11240, 112400M, International Society for Optics and Photonics (2020).

[12] Agrawal, S., Fadden, C., Dangi, A., Yang, X., Albahrani, H., Frings, N., Heidari Zadi, S., and Kothapalli, S.-R., "Light-emitting-diode-based multispectral photoacoustic computed tomography system," Sensors 19(22), 4861 (2019).

[13] Bulsink, R., Kuniyil Ajith Singh, M., Xavierselvan, M., Mallidi, S., Steenbergen, W., and Francis, K. J., "Oxygen saturation imaging using led-based photoacoustic system," Sensors 21(1), 283 (2021).

[14] Francis, K. J., Boink, Y. E., Dantuma, M., Singh, M. K. A., Manohar, S., and Steenbergen, W., "Tomographic imaging with an ultrasound and led-based photoacoustic system," Biomedical Optics Express 11(4), 2152-2165 (2020).

[15] Francis, K. J., Boink, Y. E., Dantuma, M., Singh, M. K. A., Manohar, S., and Steenbergen, W., "Light emitting diodes based photoacoustic and ultrasound tomography: Imaging aspects and applications," in [LED-Based Photoacoustic Imaging], 245-266, Springer (2020).

[16] Francis, K. J., Chinni, B., Channappayya, S. S., Pachamuthu, R., Dogra, V. S., and Rao, N., "Multiview spatial compounding using lens-based photoacoustic imaging system," Photoacoustics 13, 85-94 (2019).

[17] Francis, K. J., Booijink, R., Bansal, R., and Steenbergen, W., "Tomographic ultrasound and led-based photoacoustic system for preclinical imaging," Sensors 20(10), 2793 (2020). 